\title{
A Prospective Multicenter Study of the Chinese Scoring System for Hepatitis B Liver Failure
}

\author{
Zhe-bin Wu \\ the Third Affiliated Hospital of Sun Yat-sen University \\ Ke Wei-min \\ the Third Affiliated Hospital of Sun Yat-sen University \\ Lin Bing-liang \\ the Third Affiliated Hospital of Sun Yat-sen University \\ Peng Liang \\ the Third Affiliated Hospital of Sun Yat-sen University \\ Chen Zhi \\ The First Affiliated Hospital of Zhejiang University \\ Zhang Xin-xin \\ Shanghai Jiao Tong University Medical School Affiliated Ruijin Hospital \\ Tan De-ming \\ Xiangya Hospital Central South University

\section{Ren Wan-hua} \\ Shandong Provincial Hospital \\ Wang Kai \\ Shandong University Qilu Hospital \\ Yan Xue-bin \\ The Affiliated Hospital of Xuzhou Medical University \\ Zhi-liang Gao ( $\square$ zhilianggao@21cn.com ) \\ the Third Affiliated Hospital of Sun Yat-sen University
}

\section{Research Article}

Keywords: Hepatitis B, Liver failure, Scoring system, End-stage liver disease model

Posted Date: March 15th, 2021

DOI: https://doi.org/10.21203/rs.3.rs-288395/v1

License: @ (i) This work is licensed under a Creative Commons Attribution 4.0 International License. Read Full License 


\section{Abstract}

Objective: To evaluate the clinical utility of a Chinese scoring system for hepatitis B liver failure in a prospective and multicenter study.

Methods: Clinical data for 1143 patients with hepatitis B liver failure who had been followed up for a minimum of 6 months were collected from seven liver disease centers across China. The disease severity and prognosis for the patients were predicted using the Chinese scoring system and compared to those predicted with the model for end-stage liver disease (MELD) score, MELD-Na score, and Child-Turcotte Pugh (CTP) score.

Results: The Chinese scoring system was more effective at predicting the outcomes of survival and mortality than the MELD score. In the peak disease stage, the area under the Receiver Operating Characteristic curve for the Chinese scoring system was 0.954 , significantly higher than that $(0.896)$ for the MELD scoring system $(P<0.001)$. The positive prediction at 30,90 , and 180 days with the Chinese scoring system was 0.764 (95\% confidence interval [Cl]: 0.714-0.808), 0.731 (95\% Cl: 0.694-0.769), and 0.724 (95\% Cl: 0.679-0.765), also significantly higher than that with the MELD, MELD-Na, and CTP scores $(P<0.001)$. In addition, the Chinese scoring system was superior to the MELD, MELD-Na, and CTP scores $(P<0.001)$ at predicting the prognosis of patients with hepatitis B liver failure at both 30 and 180 days.

Conclusion: The Chinese scoring system demonstrated superior performance to the three established scoring systems in assessing the severity and outcomes of hepatitis B liver failure in this cohort.

\section{Introduction}

Liver failure is a severe complication in which the liver fails to perform basic functions due to severe or massive liver injury, which also disrupts the vital functions of other organs and systems. It typically manifests as jaundice, hepatic encephalopathy, and coagulopathy ${ }^{[1-2]}$. The mortality rate of liver failure may be as high as $70 \%{ }^{[3]}$. It is critical that hepatologists are able to assess and predict the outcomes of liver failure accurately and early in patients, especially to determine if and when a liver transplantation is required. Dynamic changes in the conditions of a patient should also be closely monitored and followed, as a timely prevention and treatment of complications significantly impacts the clinical outcomes. The model for end-stage liver disease (MELD), MELD-Na, and Child-Turcotte Pugh (CTP) scores are three established systems used to numerally grade the severity of liver failure in patients, which can reveal the probability for

favorable or non-favorable outcomes ${ }^{[4,5]}$. All three model systems were established by assessing patients with liver failure mainly caused by hepatitis $\mathrm{C}$ virus (HCV) infection and alcoholic liver disease. However, liver failure caused by hepatitis $B$ virus (HBV) infection is characterized by acute onset, severe liver injury, and high mortality, with distinct pathogenesis and clinical characteristics from those caused by HCV and alcohol. The three model systems can be expanded or modified to better grade HBV liver failure. We previously established a new scoring system known as the Chinese scoring system and its performance and utility were evaluated for patients with hepatitis B liver failure in a retrospective study ${ }^{[6-9]}$. To further investigate the utility of this new scoring system in grading the severity of hepatitis B liver failure and predicting outcomes, we conducted this prospective study at seven liver disease centers across China.

\section{Ethics Statement}

All procedures performed in studies involving human participants were approved by Ethics Committee of the Third Affiliated Hospital of Sun Yat-sen University. All procedures performed in studies involving human participants were in accordance with the ethical standards of the institutional and/or national research committee and with the 1964 Helsinki declaration and its later amendments or comparable ethical standards. This article does not contain any studies with animals performed by any of the authors. Informed consent was obtained from all individual participants included in the study.

\section{Materials And Methods}




\section{Patients}

1.1 Enrollment Between October 2013 and June 2016, a total of 1143 chronic hepatitis B inpatients with liver failure (acuteon-chronic liver failure, ACLF) were enrolled from seven liver disease centers across China (infection department of the Third Affiliated Hospital of Sun Yat sen University, infection department of the First Affiliated Hospital of Zhejiang University, Shanghai Ruijin Hospital, Xiangya Hospital of Central South University, Qilu Hospital of Shandong University, Shandong Provincial Hospital, and Xuzhou Medical College). Among them, 936 were men, 207 were women, and the average age was $43.4 \pm 12.6$ (ranged from 18 to 65 ). The patients were followed up for $>6$ months, during which 464 died and 680 survived.

HBV-related ACLF diagnosis for all patients followed the criteria established by the 18th Asia-Pacific Association of Liver Research consensus on chronic-acute liver failure ${ }^{[10]}$. The criteria include a history of chronic hepatitis $B$ and acute flare-up of liver injury with clinical manifestations of jaundice (total bilirubin $\geq 85 \mathrm{umol} / \mathrm{L}$ ) and coagulation disorder (prothrombin time international standardized ratio $\geq 1.5$ ), ascites and / or hepatic encephalopathy within 4 weeks.

1.2 Inclusion criteria Male or female patients aged 18-60; HBsAg-positive history > 6 months, HBV DNA-positive, HBeAgpositive or negative; persistent hepatitis symptoms of fatigue, anorexia, abdominal distention, or yellow urine; gradual aggravation of jaundice over a short period of time; total serum bilirubin $\geq 85 \mathrm{umol} / \mathrm{L}$ or daily elevation $\geq 17.1 \mathrm{umol} / \mathrm{L}$; abnormal coagulation function, and international standardized ratio of prothrombin time $\geq 1.5$.

1.3 Exclusion criteria Patients were excluded if they: (1) had other hepatitis virus infection; (2) had human immunodeficiency virus infection, biliary, alcoholic liver, or autoimmune liver diseases; drug poisoning, liver tumors, or were undergoing liver transplantation, renal insufficiency, or long-term anticoagulant therapy related to renal diseases.

2. Observations and follow-up endpoints Clinical information and test findings for all patients at and after admission were collected weekly. The clinical information mainly consisted of the stages of hepatic encephalopathy. Laboratory findings included serum total bilirubin, albumin, creatinine, prothrombin time, prothrombin time international normalized ratio (INR), serum sodium ion concentration, liver size (B-mode ultrasound measurement), ascites, and pleural effusion (B-ultrasound measurement), as well as infection (peripheral white blood cell count, neutrophil ratio, and chest inflammation images). The end point of 180 days of follow-up was used to determine the survival rate. The death count included patients that rejected rescue treatment and were discharged from the hospital as well as patients that died during the hospital stay or within 180 days of follow-up.

\section{Score calculation}

3.1 MELD score Since all subjects had hepatitis B-related liver failure, the MELD score was calculated as 3.8xloge (serum bilirubin umol/L×0.058) + 1.2×loge (prothrombin time INR) + 9.6×loge (serum creatinine umol/L×0.011) $+6.4^{[6]}$.

3.2 MELD-Na This score was calculated as MELD + 1.59×(135- $\left.\mathrm{Na}^{+}\right)$, wherein serum $\mathrm{Na}^{+}$levels $\geq 135 \mathrm{mmol} / \mathrm{L}$ were treated as $135 \mathrm{mmol} / \mathrm{L}, \leq 120 \mathrm{mmol} / \mathrm{L}$ as $120 \mathrm{mmol} / \mathrm{L}$, and between $120 \sim 135 \mathrm{mmol} / \mathrm{L}$ as the specific value $\mathrm{e}^{[7]}$.

3.3 CTP score This score was calculated using the scoring standards for five indexes, i.e. grade of hepatic encephalopathy, ascites, total bilirubin, albumin, and prolonged prothrombin time. A score of 1, 2, or 3 was assigned to each index to reflect the severity of each condition, and the CTP score was the sum of the five indexes ${ }^{[8]}$.

3.4 Chinese scoring system This system consists of seven clinical indicators: prothrombin activity, serum creatinine, hepatic encephalopathy, serum total bilirubin, liver size (B-ultrasonic measurement), ascites/pleural fluid (B-ultrasonic measurement) volume, and infection (peripheral blood leukocyte count, neutrophil ratio, and chest inflammation image). A score of $1,2,3$, or 4 was assigned to each indicator to reflect the severity, and the sum of the seven indicator scores was used (see Table 1 for details). 


\section{Statistical analysis}

(1) All numeral data were expressed as mean \pm standard deviation $( \pm s)$ and the difference was computed using the $f$ test.

(2) The count data were expressed as percentage (\%), and the difference was assessed with the $\chi^{2}$ test. (3) Assessments of the short-term and long-term prognoses for patients with hepatitis B liver failure using the Chinese scoring system, MELD score, MELD-Na score, and CTP score were compared using receiver operating characteristic (ROC) curves and the ROC area under the curve (AUC). AUC $>0.7$ was deemed to be of clinical utility and $>0.8$ to be of good prediction accuracy. The AUC values were compared using normal $\mathrm{Z}$ tests and the ROC curve sensitivity and specificity were used to determine the best cut-off values for the score and the Youden index. SPSS 18.0 statistical software was used for all analyses. P $<0.05$ was considered statistically significant.

\section{Results}

1. Demographic features and groups. A total of 1143 patients with hepatitis $B$ liver failure were enrolled in this study. Among them, 936 were men and 207 were women, and the mean age was $43.4 \pm 12.6$ years old (ranged between 18 and 65 ). A total of 463 patients died while the remaining 680 survived (Table 2).

2. The cutoff value and distribution range for the Chinese scoring system and the MELD score to separate the patients that survived from those that did not. The cutoff value of 12.14 with the Chinese scoring system separated $83.65 \%$ of patients in the survival and death groups, i.e. 83.65\% patients in the survival group scored between 3.68 and 12.14 points, while $83.65 \%$ patients in the death group scored between 12.14 and 22.13 points. With the MELD score, the cutoff value was $31.78 ; 64.52 \%$ of patients in the survival group scored between 20.37 and 31.78 points, and $64.52 \%$ of patients in the death group scored between 31.78 and 49.88 points (see Figure 1 and Figure 2, $P<0.01$ ).

\section{Comparison of the performance to grade the severity at the peak disease with the Chinese scoring system and MELD} scoring system The ROC curve was plotted using the total scores. The area under the ROC curve for the Chinese scoring system was 0.954 (95\% confidence interval [Cl]: 0.943-0.971) and the standard error was 0.013 , with $P<0.001$, suggesting good prediction performance. The area under the ROC curve for the MELD score was 0.896 (95\% Cl: 0.881-0.924), indicating less effectiveness than the Chinese score system (see Figure 3 and table 3 ).

4. The ability to predict and differentiate disease outcomes at different timeswith different scoring systems The predictive values for the outcomes on days 30,90, and 180 after discharge using the Chinese scoring system were 0.764 (95\% Cl: $0.714-0.808), 0.731$ (95\% Cl: 0.694-0.769), and 0.724 (95\% Cl: 0.679-0.765), respectively, which were significantly higher than those for the other three scoring systems (see Table $4, \mathrm{P}<0.001$ ).

5. Comparison of the performance predicting short-term (30 days) prognosis for patients with hepatitis B liver failure using the Chinese scoring system, MELD score, MELD Na score, and CTP score The area under the ROC curve with the total score from the Chinese scoring system was 0.783 (95\% Cl: 0.732-0.865) and the standard error was 0.026 , with $\mathrm{P}<0.001$, suggesting good predictive performance. The areas under the ROC curves for the MELD score, MELD Na score, and CTP score were 0.706 (95\% Cl: 0.623-0.778), 0.716 (95\% Cl: 0.644-0.779) and 0.686 (95\% Cl: 0.623-0.767), respectively, which were significantly lower than that for the Chinese scoring system (see Figure 4 and table 5 ).

\section{Comparison of the performance predicting long-term (180 days) prognosis for patients with hepatitis B liver failure using} Chinese scoring system, MELD score, MELD-Na score, and CTP score The area under the ROC curve with the total score from the Chinese scoring system was 0.748 (95\% Cl: 0.692-0.837) and the standard error was 0.022 , with $P<0.001$, suggesting good predictive performance. The areas under the ROC curves for the MELD score, MELD Na score, and CTP score were 0.657 (95\% Cl: 0.588-0.725), 0.676 (95\% Cl: 0.605-0.743), 0.682 (95\% Cl: 0.621-0.752), respectively, which were significantly lower than that for the Chinese scoring system (see Figure 5 and table 6). 


\section{Discussion}

MELD, MELD-Na, and CTP scores have been established to address the need to accurately identify patients with liver failure who require liver transplants. All three systems are widely accepted in the clinical setting. However, these models were constructed using clinical data largely from patients with liver failure in Europe and the United States where the major etiologies for liver failure are alcohol, drugs, hepatitis $C$, and cholestasis. In contrast, hepatitis B represents the dominant etiology for liver failure in China and is responsible for $85 \%$ of liver failure cases in the country ${ }^{[11]}$. Therefore, these three models ${ }^{[12-13]}$ may not completely fit Chinese patients with liver failure. As noted by Lin Xianfeng et al ${ }^{[14]}$, the MELD score failed to accurately predict the prognosis of 327 Chinese patients with hepatitis B liver failure. One factor that may have affected this result is that patients with HBV liver failure do not respond well to comprehensive treatment and often need an artificial liver, liver transplant, or other treatment urgently. The other factor is that chronic HBV infection may exhaust patients' immunity, leading to severe complications. A new system that takes these factors into consideration is required ${ }^{[15]}$.

CTP has been widely employed in managing patients with liver diseases because of its simplicity and ease of calculation. However, it is often hindered by a lack of consistency as CTP is mainly based on albumin level and prothrombin time, both of which can be masked if exogenous albumin and plasma products are infused. In addition, hepatic encephalopathy and ascites measurement can be subjective and variable. CTP only consists of three grades with a narrow score range of 5-15, which limits the ability to differentiate. Patients with the same score may differ greatly in the severity of liver disease.

Although the MELD and MELD-Na scoring systems are recognized as easy to use, reproducible, and accurate in clinical applications, they bear an inherent limitation because they only include bilirubin, coagulation function, renal function, and etiology, while excluding the complications of liver failure (infection, hemorrhage, hepatic encephalopathy, brain edema, or ascites). The Na concentration in the MELD-Na scoring system can be easily affected by insufficient intake, diuretics, or sodium pump failure ${ }^{[16-21]}$. A study conducted in China ${ }^{[22]}$ showed that the AUC of MELD and MELD-Na scores for the 12week outcomes of patients with hepatitis B-related liver failure was only $0.731,0.735$, and 0.773 , respectively, and the sensitivity was below 0.7 .

This prospective study conducted at seven large liver disease centers across China showed that the Chinese scoring system better distinguished patients that survived from those who did not, in comparison to MELD $(P<0.001)$. The area under the ROC curve for the Chinese scoring system was 0.954 in the peak disease stage, suggesting a stronger ability to grade the severity of liver failure. The AUC for the MELD scoring system was 0.896 , which was relatively high, but significantly lower than that for the new scoring system $(P<0.001)$. We also found that the efficiency in predicting outcomes on the 30th, 90th, and 180th day after discharge with the Chinese scoring system was significantly higher than that with MELD, MELD Na, and CTP $(P<0.001)$.

Massive or submassive liver injury following a flare-up of HBV replication or HBV reactivation compromises synthetic, metabolic, and detoxification functions in the liver, leading to reduced albumin levels and ascites, hyperbilirubinemia, decreased PTA(Prothrombin activity), increased international standard ratio of prothrombin (INR), and hepatic encephalopathy. In addition, a severely injured liver may also hurt Kupffer cell function and reduce complement levels that may limit the anti-infective capacity. The accumulation of toxins and a decrease in renal blood flow may facilitate hyperbilirubinemia, renal function insufficiency, or even renal failure. In theory, the severity of liver failure among different individuals can be indicated by biomarkers that reflect critical alterations in the pathophysiology of liver failure. Thus, we formulated this new scoring system to improve severity grading and outcome prediction. A total of seven clinical indicators of hepatitis B liver failure are included in the Chinese scoring system, consisting of not only objective indicators such as $\mathrm{Cr}$, total bilirubin, PTA, and liver size, but also the complications of hepatic encephalopathy, ascites with pleural effusion, and pulmonary infection, which reflect the pathophysiological changes resulting from hepatitis $B$ liver failure. The score boundary point was confirmed using the interactive chi square test, guided by the principle that emphasizes simplicity and clarity. Each index is graded between 0-4 points, allowing a high degree of differentiation that increases prediction accuracy.

Page 5/13 
This new scoring system utilizes common clinical indicators, which can be routinely ordered to allow easy and wide applications.

This new scoring system demonstrated superior performance in grading the severity and predicting the outcomes of HBV liver failure in this cohort compared to the three established scoring systems, representing progress in improving the management of HBV liver failure. However, our findings will need further verification in larger cohorts that include not only patients with HBV liver failure, but also those with liver failure with other etiologies.

\section{Declarations}

Conflict of interest: No any editorial or financial conflict of interest exists.

Sources of financial or support for the manuscript: the National Science and

Technology Major Project (2018ZX10302204-002)

Author contributions: Wu Zhebin performed case summary and statistical analysis, and wrote the manuscript; Ke Weimin designed this scoring system; Lin Bing-liang and Peng Liang were responsible for statistical analysis;Chen Zhi, Zhang Xinxin, Tan De-ming, Ren Wan-hua, Wang Kai and Yan Xue-bin were responsible for case enrollment and data collection in other subcenters; Zhiliang Gao was responsible for research design and the whole quality of the study.

\section{References}

1. Zou Zhengsheng, Chen Jumei, Xin Shaojie et al. Analysis of the clinical characteristics of 565 cases of severe viral hepatitis [J]. Chinese Journal of Hepatology,2001,9(4):247凶248\

2. Hepatic Failure and Artificial Liver Group, Infectious Disease Branch, Chinese Medical Association, Severe Liver Disease and Artificial Liver Group, Hepatology Branch, Chinese Medical Association. Guidelines for Diagnosis and Treatment of Liver Failure [J] .Chinese Journal of Hepatology,2006,14(9):643₫646》

3. Vizzutti F, Arena U, Laffi G, et al. Acute on chronic liver failure:from pathophysiology to clinical management[J]. Trends Anaesth Crit Care. 2013ه 3:122-129.

4. Christensen E. Prognostic models including the Child-Pugh, MELD and Mayo risk scores: where are we and where should we go?[J]. J Hepatol, 2004,41(2):344-350》

5. Kamath PS, Wiesner RH, Malinchoc M, et al. A model to predict survival in patients with end-stage liver disease[J].Hepatology, 2001, 33(2):464-470区

6. Yu-Bao Zheng,Zhan Lian Huang,Zhe Bin Wu,et al.Dynamic Changes of Clinical Features that Predict the Prognosis of Acute-on-Chronic Hepatitis B Liver Failure:A Retrospective Cohort Study[J]. International Journal of Medical Sciences.2013,Vol.10,10(12):1658-1664.

7. Yubao Zheng, Dongying Xie, YuRong Gu, et al. Development of a sensitive prognostic scoring system for the evaluation of severity of acute-on-chronic hepatitis B liver failure: A retrospective cohort study[J]. Clin Invest Med. 2012 Apr 1;35(2):E75-85.

8. Yu-Bao Zheng, Shi-Bin Xie, Dong-Ying Xie, et al. A Novel prognostic scoring system to predict 3-month mortality risk in patients with acute-on-chronic liver failure in hepatitis B: a retrospective cohort study[J]. Hepatology International, 2012 , 6(4):727-734.

9. Li Xinyan, Lei Ruixiang, Ke Weimin, et al. Simple scoring system for the severity of chronic hepatitis A with chronic liver failure. Chinese Medical Journal, 2009,89(47):3353-3355.

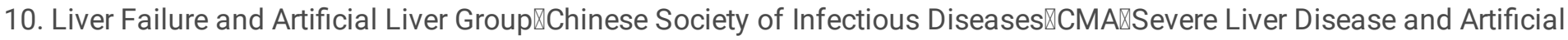

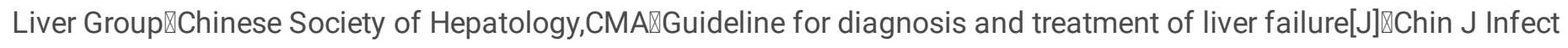


Dis,2012,5®321-327》

11. Zhang Dongqing, Chen Li, Gan Qiaorong, etc. Analysis of prognostic factors in patients with hepatitis B-related chronic and acute liver failure [J]. Journal of Clinical Hepatobiliary Disease $2012,28 \otimes 10 \bigotimes: 740 \bigotimes 743 \rrbracket$

12. RufAE\Kremers WK,Chavez LL『et a1囚Addition of serum sodium into the MELD score predicts waiting list mortality better than MELD alone[J]囚Liver Transpl, 2005囚11:336-343》

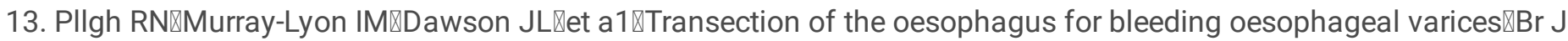
Surg $₫ 1973 \rrbracket 60 \rrbracket 646-649 \rrbracket$

14. Lin Xianfeng, Li Lingfei, Yu Yue, etc. The predictive value of MELD-related scoring system for the prognosis of patients with chronic hepatitis-related chronic and acute liver failure [J / CD]. Chinese Journal of Clinicians: Electronic Edition $\nabla$ $2012,6 \rrbracket 198 \otimes 5853 \rrbracket 5857 \rrbracket$

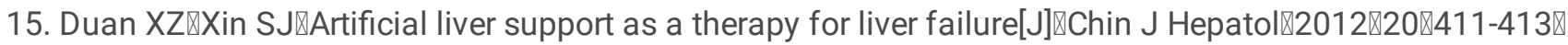

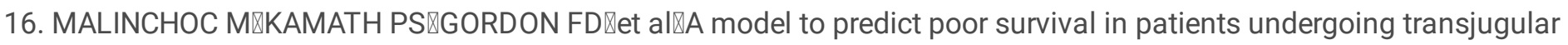
intrahepatic portosystemic shunt[J]区hepatology $\llbracket 2000 \rrbracket 31(4) \llbracket 864 \llbracket 871 \rrbracket$

17. RufAE『Kremers WK,Chavez LL『et a1『Addition of serum sodium into the MELD score predicts waiting list mortality better than MELD alone[J]囚Liver Transpl, 2005, 11囚336-343囚

18. Kim JM『Kwon CH囚Joh JW\et al. Can themodel for end-stage liver disease score replace the indocyanine green clearance test in the selection of right hemihepatectomy in Child-Pugh class A.Ann Surg Treat Res,2014『86『3®:122-129.

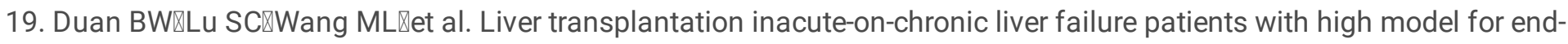
stage liver disease $\triangle M E L D \bigotimes$ scores:a single center experience of 100 consecutive cases. J Surg Res,2013,183 (2):936943.

20. Basile-Filho AđNicolini EA囚Auxiliadora-Martins M】et al.Comparison of acutephysiology and chronic health evaluation II death risk $₫$ Child-Pugh $₫$ Charlsonखand model for end-stage liver disease indexes to predict early mortality after liver transplantation. Transplant Proc®2011『43(5):1660-1664.

21. Biselli $M$, Dall'agata $M$, Gramenzi $A$, et al. A new prognostic model to predict dropout from the waiting list in cirrhotic candidates for liver transplantation with MELD score<18. Liver Int 2015;35:18-191.

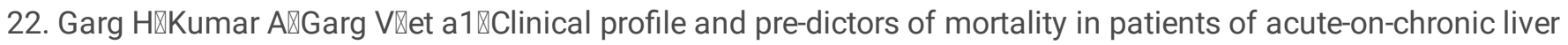
failure[J] $₫$ Dig Liver Dis $₫ 2012 \rrbracket 44(2) \bigotimes 166-171 \rrbracket$

\section{Tables}


Table 1

Assigned scores of the Chinise scoring system

\begin{tabular}{|c|c|c|c|c|c|c|c|}
\hline $\begin{array}{l}\text { Scoring } \\
\text { (score) }\end{array}$ & $\begin{array}{l}\text { Hepatic } \\
\text { encephalopathy } \\
\text { (Stage) }\end{array}$ & $\begin{array}{l}\text { Total } \\
\text { bilirubin } \\
\text { ( } \mu \mathrm{mol} / \mathrm{L})\end{array}$ & $\begin{array}{l}\text { The } \\
\text { maximum } \\
\text { depth of } \\
\text { ascites }(\mathrm{mm})\end{array}$ & $\begin{array}{l}\text { Activity } \\
\text { of } \\
\text { PT(\%) }\end{array}$ & $\begin{array}{l}\text { Right hepatic } \\
\text { oblique } \\
\text { diameter/thickness } \\
(\mathrm{mm})\end{array}$ & $\begin{array}{l}\text { Creatinine } \\
(\mu \mathrm{mol} / \mathrm{L})\end{array}$ & $\begin{array}{l}\text { Infection } \\
\text { (depend on } \\
\text { WBC } 10^{9} / \mathrm{L} \text { ) }\end{array}$ \\
\hline 1 & ] & $\begin{array}{l}\geq 10 \sim \\
20 U L N\end{array}$ & $>0 \sim 40$ & $\begin{array}{l}30 \sim< \\
40\end{array}$ & $\begin{array}{l}\text { Oblique diamete } \geq \\
120 \text { or Thickness } \\
\geq 110\end{array}$ & $\begin{array}{l}>1.0 \sim \\
1.1 \mathrm{ULN}\end{array}$ & $\begin{array}{l}\text { WBC }>10 \sim \\
15 \\
\text { Or N }> \\
70 \% \sim 80 \%\end{array}$ \\
\hline 2 & प & $\begin{array}{l}>20 \sim \\
30 U L N\end{array}$ & $>40 \sim 80$ & $\begin{array}{l}20 \sim< \\
30\end{array}$ & $\begin{array}{l}\text { Oblique diameter } \\
110 \sim<120 \text { or } \\
\text { Thickness } 100 \sim< \\
110\end{array}$ & $\begin{array}{l}>1.1 \sim \\
1.2 \mathrm{ULN}\end{array}$ & $\begin{array}{l}\text { WBC }>15 \sim \\
20 \\
\text { Or N> } \\
80 \% \sim 90 \%\end{array}$ \\
\hline 3 & ] & $\begin{array}{l}>30 \sim \sim \\
40 U L N\end{array}$ & $>80$ & $\begin{array}{l}10 \sim< \\
20\end{array}$ & $\begin{array}{l}\text { Oblique } \\
\text { diameter100 < } \\
110 \text { or Thickness } \\
90 \sim<100\end{array}$ & $\begin{array}{l}>1.2 \sim \\
1.3 U L N\end{array}$ & $\begin{array}{l}W B C>20 \\
\text { Or } N>90 \%\end{array}$ \\
\hline 4 & प & $>40 U L N$ & $\begin{array}{l}>80+\text { one or } \\
\text { both } \\
\text { side pleural } \\
\text { fluid }\end{array}$ & $<10$ & $\begin{array}{l}\text { Oblique diameter < } \\
100 \\
\text { or Thickness }<90\end{array}$ & $>1.3 \mathrm{ULN}$ & $\begin{array}{l}\text { Inflammation } \\
\text { manifestation } \\
\text { of lung }\end{array}$ \\
\hline
\end{tabular}

पसULN: Upper limit of normal.

\Score of ascites: Regardless of the amount of ascites, the presence of unilateral or bilateral pleural fluid is counted as 4 points.

هLiver size scoring: If the right liver oblique diameter and thickness are measured at the same time on the ultrasound image, the high-scoring scoring results are taken.

QInfection score: The standard of 4 points is the imaging changes of pulmonary inflammation, regardless of the white blood cells And neutrophil counted as 4 points. 
Table 2

Baseline characteristics of included patients at admission

\begin{tabular}{|c|c|c|c|c|}
\hline Parameters & Death group $(n=463)$ & Survival group $(n=680)$ & $t / x 2$ value & $\mathbf{P}$ \\
\hline Age (year) & $45.38 \pm 11.73$ & $40.06 \pm 10.47$ & 1.84 & 0.12 \\
\hline Males (\%) & $364 / 99$ & $572 / 108$ & 1.99 & 0.06 \\
\hline WBC (×109/L) & $7.82 \pm 3.54$ & $6.98 \pm 3.72$ & 1.25 & 0.38 \\
\hline ALT (U/L) & $568.47 \pm 376.94$ & $629.76 \pm 504.57$ & 1.56 & 0.28 \\
\hline Albumin $(\mathrm{g} / \mathrm{L})$ & $31.64 \pm 3.56$ & $32.85 \pm 4.02$ & 0.98 & 0.47 \\
\hline TB (Imol/L) & $428.75 \pm 284.63$ & $353.96 \pm 249.66$ & 3.45 & $<0.001$ \\
\hline PTA (\%) & $29.86 \pm 11.58$ & $34.38 \pm 10.85$ & 4.78 & $<0.001$ \\
\hline INR & $3.41 \pm 0.68$ & $2.83 \pm 0.62$ & 4.27 & $<0.001$ \\
\hline $\mathrm{BUN} /(\mathrm{mmol} / \mathrm{L})$ & $5.84 \pm 3.68$ & $5.22 \pm 3.76$ & 1.96 & 0.08 \\
\hline $\mathrm{CR} /(\mu \mathrm{mol} / \mathrm{L})$ & $82.37 \pm 54.16$ & $67.47 \pm 44.85$ & 1.92 & 0.09 \\
\hline HBV DNA,median(range), Log10 IU/ml & $6.89(4.30-8.86)$ & $6.62(5.02-8.64)$ & 1.89 & 0.16 \\
\hline Without cirrhosis, n(\%) & 296(63.93) & 258(37.94) & 8.94 & $<0.001$ \\
\hline Encephalopathy (\%) & 16.58 & 9.73 & 10.76 & $<0.001$ \\
\hline HRS (\%) & 8.23 & 5.13 & 2.95 & $<0.001$ \\
\hline $\operatorname{SBP}(\%)$ & 54.32 & 31.06 & 6.83 & $<0.001$ \\
\hline Ascites (\%) & 67.43 & 48.14 & 3.12 & $<0.001$ \\
\hline MELD & $40.52 \pm 6.83$ & $26.84 \pm 6.18$ & 16.83 & $<0.001$ \\
\hline MELD-Na & $44.76 \pm 7.83$ & $28.62 \pm 5.93$ & 19.05 & $<0.001$ \\
\hline CTP & $11.87 \pm 1.68$ & $10.56 \pm 1.47$ & 2.01 & 0.04 \\
\hline Chinise scoring system & $17.53 \pm 3.92$ & $8.46 \pm 3.28$ & 22.18 & $<0.001$ \\
\hline
\end{tabular}

Table 3

Comparison of the performance to grade the severity at the peak disease by Chinese scoring system and MELD scoring system

\begin{tabular}{|lll|}
\hline & AUROC $(95 \% \mathrm{Cl})$ & p value vs. CSS-LFB \\
\hline CSS-LFB & $0.954(0.943-0.971)$ & \\
\hline MELDs & $0.896(0.881-0.924)$ & $<0.001$ \\
\hline
\end{tabular}


Table 4

The ability to predict and differentiate disease outcomes at different times with different scoring systems

\begin{tabular}{|lllll|}
\hline & $\begin{array}{l}\text { CSS-LFB } \\
\text { C-index }(95 \% \mathrm{Cl})\end{array}$ & $\begin{array}{l}\text { MELD } \\
\text { C-index }(95 \% \mathrm{Cl})\end{array}$ & $\begin{array}{l}\text { MELD-Na } \\
\text { C-index (95\% Cl) }\end{array}$ & $\begin{array}{l}\text { Child-Pugh } \\
\text { C-index (95\% Cl) }\end{array}$ \\
\hline $\begin{array}{l}\text { 30-day mortality } \\
\text { p value vs.CSS }\end{array}$ & $0.764(0.714-0.808)$ & $\begin{array}{l}0.684(0.632-0.743) \\
<0.001\end{array}$ & $\begin{array}{l}0.685(0.634-0.738) \\
<0.001\end{array}$ & $\begin{array}{l}0.664(0.612-0.725) \\
<0.001\end{array}$ \\
$\begin{array}{l}\text { 90-day mortality } \\
\text { p value vs.CSS }\end{array}$ & $0.731(0.694-0.769)$ & $\begin{array}{l}0.657(0.612-0.713) \\
<0.001\end{array}$ & $\begin{array}{l}0.662(0.616-0.712) \\
<0.001\end{array}$ & $\begin{array}{l}0.656(0.607-0.704) \\
<0.001\end{array}$ \\
$\begin{array}{l}\text { 180-day mortality } \\
\text { p value vs.CSS }\end{array}$ & $0.724(0.679-0.765)$ & $\begin{array}{l}0.648(0.604-0.698) \\
<0.001\end{array}$ & $\begin{array}{l}0.655(0.607-0.699) \\
<0.001\end{array}$ & $\begin{array}{l}0.642(0.593-0.691) \\
<0.001\end{array}$ \\
\hline
\end{tabular}

Table 5

Comparison of the area under the ROC curve of predicting short-term (30 days) prognosis of patients with hepatitis $B$ liver failure among Chinese scoring system, MELD score,

MELD Na scoring and CTP scoring

\begin{tabular}{|lll|}
\hline & AUROC $(95 \% \mathrm{Cl})$ & p value vs. CSS-LFB \\
\hline CSS-LFB & $0.783(0.732-0.865)$ & \\
\hline MELDs & $0.706(0.623-0.778)$ & 0.0089 \\
MELD-Nas & $0.716(0.644-0.779)$ & 0.0097 \\
CPs & $0.686(0.623-0.767)$ & 0.0075 \\
\hline
\end{tabular}

Table 6

Comparison of the area under the ROC curve of predicting long-term (180 days) prognosis of patients with hepatitis B liver failure among Chinese scoring system, MELD score,

MELD-Na scoring and CTP scoring

\begin{tabular}{|lll|}
\hline & AUROC $(95 \% \mathrm{Cl})$ & p value vs. CSS-LFB \\
\hline CSS-LFB & $0.748(0.692-0.837)$ & \\
\hline MELDs & $0.657(0.588-0.725)$ & 0.0014 \\
\hline MELD-Nas & $0.676(0.605-0.743)$ & 0.0082 \\
\hline CPs & $0.682(0.621-0.752)$ & 0.0012 \\
\hline
\end{tabular}

\section{Figures}




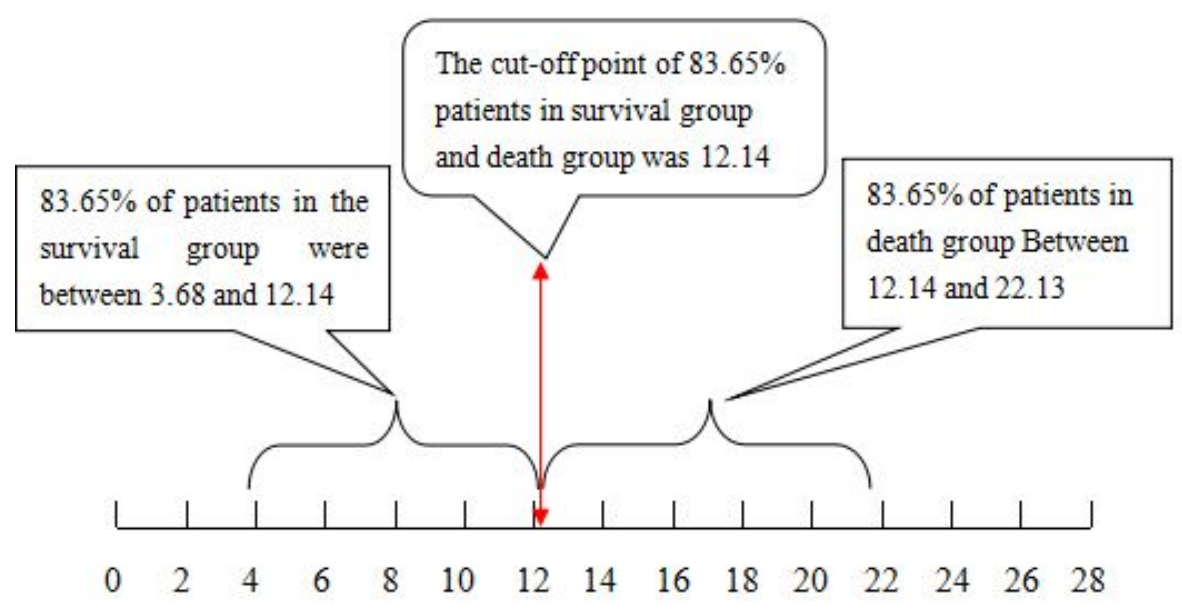

\section{Figure 1}

The cutoff value and distribution range for the Chinese scoring system to separate the patients that survived from those that did not.

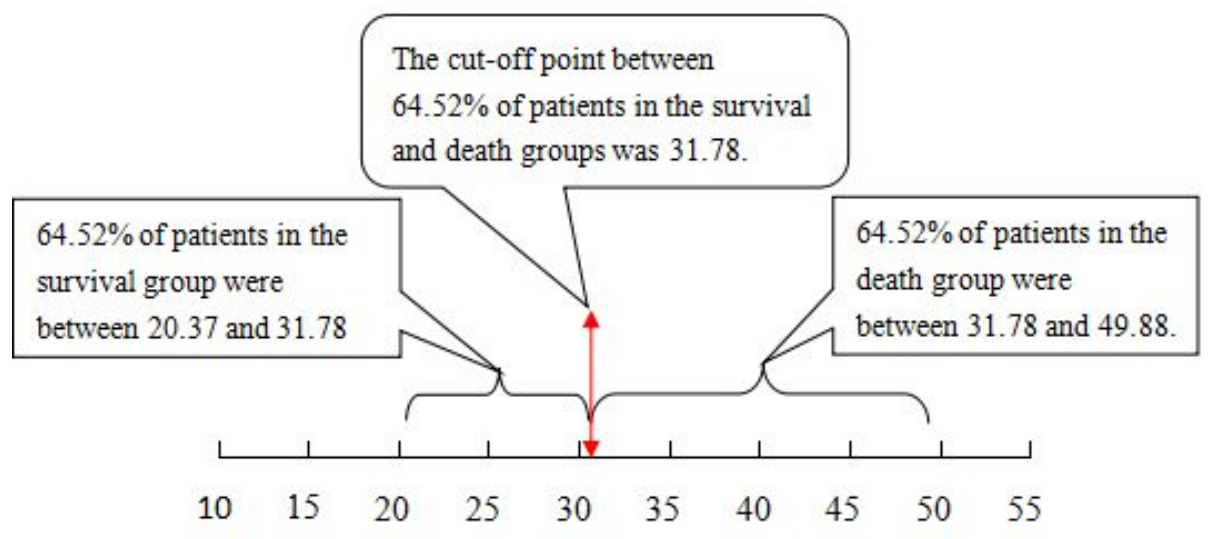

\section{Figure 2}

The cutoff value and distribution range for the MELD score to separate the patients that survived from those that did not. 


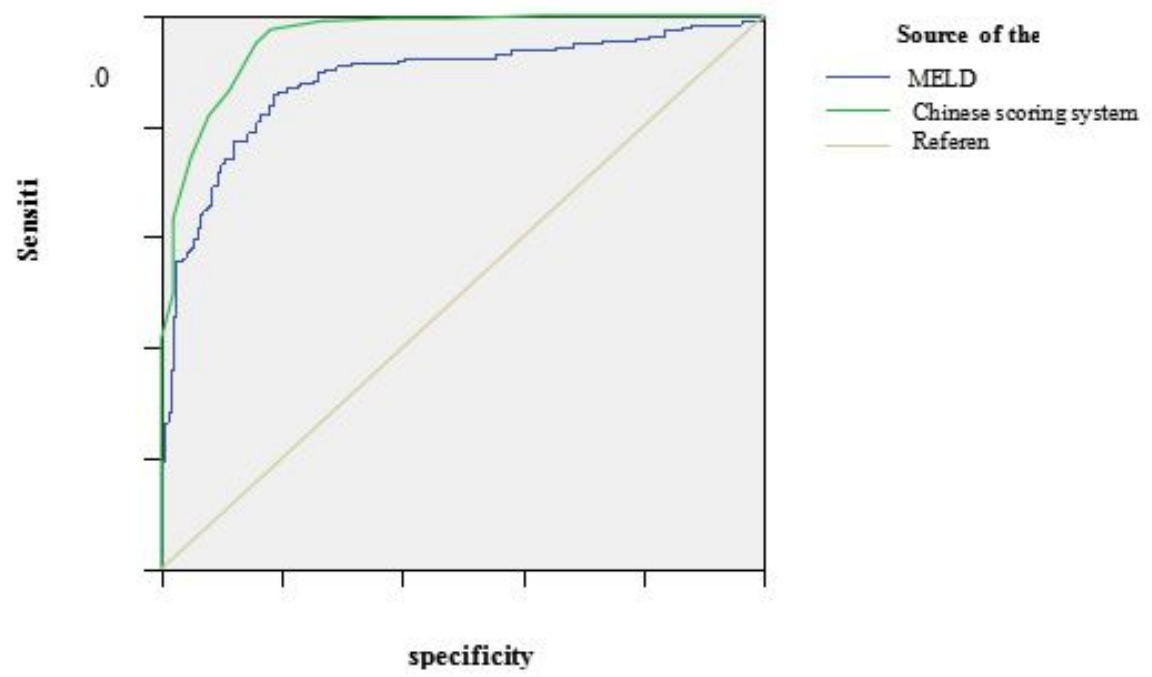

\section{Figure 3}

The ROC curve of Chinese scoring system and MELD scoring system at the peak disease.

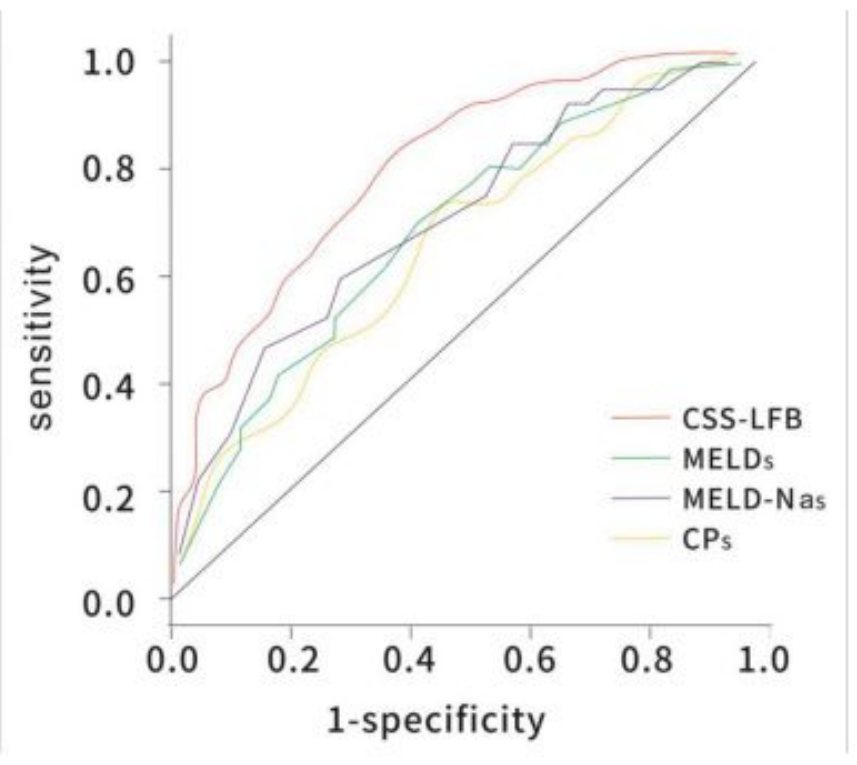

Figure 4

The ROC curve of predicting short-term (30 days) prognosis of patients with hepatitis B liver failure among Chinese scoring system, MELD score, MELD Na scoring and CTP scoring. 


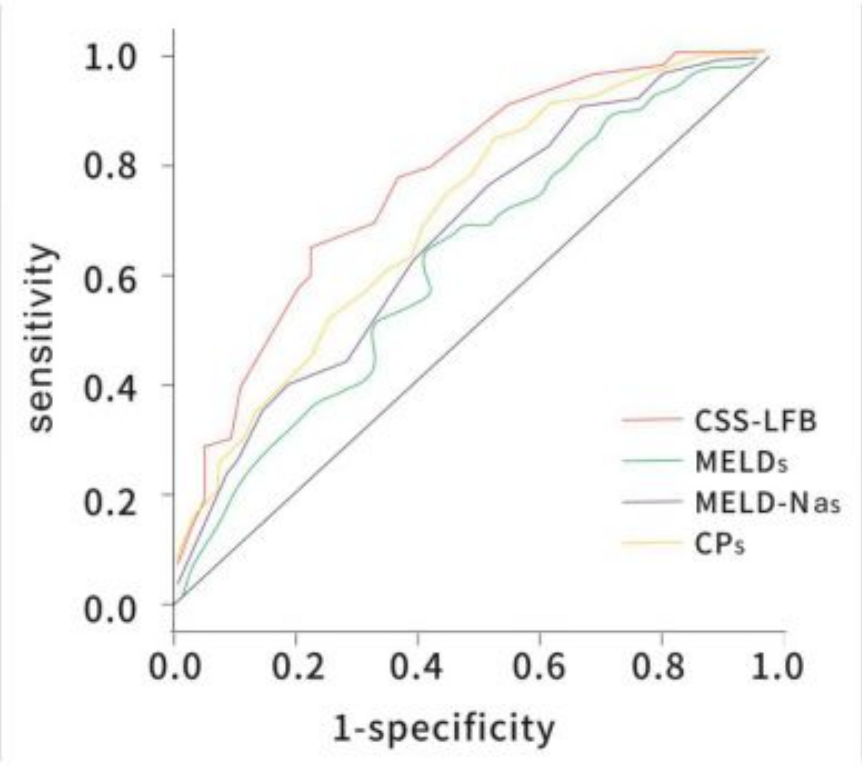

Figure 5

the ROC curve of predicting long-term (180 days) prognosis of patients with hepatitis B liver failure among Chinese scoring system, MELD score, MELD Na scoring and CTP scoring. 